\title{
Defining the Clinical, Emotional, Social, and Financial Burden of Congenital Athymia
}

Elena W. Y. Hsieh · Julie J. Kim-Chang · Sarah Kulke •

Abigail Silber · Matthew O'Hara · Cathleen Collins

Received: April 30, 2021 / Accepted: June 5, 2021 / Published online: July 2, 2021

(C) The Author(s) 2021

\section{ABSTRACT}

Introduction: Characterize the burden of illness in pediatric patients with congeñital athymia who were receiving supportive care.

Methods: This cross-sectional study of adult caregivers of patients with congenital athymia used both a quantitative survey and qualitative

E. W. Y. Hsieh

Department of Pediatrics, Section of Allergy and Immunology, Children's Hospital Colorado

University of Colorado School of Medicine, Aurora, CO, USA

E. W. Y. Hsieh

Department of Immunology and Microbiology, University of Colorado School of Medicine, Aurora, CO, USA

J. J. Kim-Chang

Division of Allergy, Immunology, and Pulmonary Medicine, Department of Pediatrics, Duke

University School of Medicine, Durham, NC, USA

S. Kulke

Enzyvant Therapeutics, Inc., Cambridge, MA, USA

A. Silber · M. O'Hara

Trinity Life Sciences, Waltham, MA, USA

C. Collins $(\bowtie)$

Rady Children's Hospital, San Diego, CA, USA

e-mail: c4collins@health.ucsd.edu

C. Collins

Department of Pediatrics, Division of Allergy Immunology, University of California San Diego, San Diego, CA, USA interviews. Caregivers of patients currently receiving supportive care responded to questions about the past 12 months and completed the parent proxy version of the Pediatric Quality of Life Inventory Generic instrument (PedsQL) for patients aged 2-4 years. For caregivers of patients who had received supportive care in the past, questions were asked about the period when they were receiving supportive care only.

Results: The sample included caregivers of 18 patients, 5 who were currently receiving supportive care and 13 who received investigational cultured human thymus tissue implantation before study enrollment and had received supportive care in the past. The impact of congenital athymia was substantial. Reports included the need to live in isolation (100\% of respondents); caregiver emotional burden such as fear of death, infection, and worries about the future (100\%); financial hardship (78\%); and the inability to meet family/friends (72\%). Patients had frequent and prolonged hospitalizations (78\%) and had high utilization of procedures, medications, and home medical supplies. Caregiver-reported PedsQL scores for patients currently receiving supportive care $(n=4)$ indicated low health-related quality of life.

Conclusions: Caregivers of patients with congenital athymia reported high clinical, emotional, social, and financial burden on patients and their families. 
Keywords: Real-world evidence; Cultured thymic tissue implantation; Thymic aplasia; Burden of illness

\section{Key Summary Points}

Why carry out this study?

Congenital athymia is an ultra-rare condition in which infants are born without a thymus and are profoundly immunodeficient; with supportive care only, the condition is fatal within the first 2-3 years of life.

Current clinical management of congenital athymia is supportive care to prevent or treat infection and immune dysregulation.

There is no published literature on the burden of illness in this ultra-rare disease; this study sought to characterize the clinical, emotional, social, and financial burden of congenital athymia on pediatric patients, their caregivers, and their families.

\section{What was learned from the study?}

Most patients experienced numerous and frequent clinical manifestations of congenital athymia, and $100 \%$ of caregivers reported multiple types of emotional, social, and/or financial burden.

When treated with supportive care only, congenital athymia exerts a substantial and wide-ranging burden on patients and their families.

\section{INTRODUCTION}

Congenital athymia is an ultra-rare (defined as prevalent in no more than 1:50,000 [1]) pediatric condition characterized by the lack of thymic development in utero. The thymus is responsible for the maturation and selection of T-cell precursors, and, as a result, pediatric patients with congenital athymia lack naïve $\mathrm{T}$ cells that normally undergo positive and negative selection in the thymus and are essential for self-tolerance and antimicrobial immunity $[2,3]$. Patients with congenital athymia are most commonly identified via low or undetectable T-cell receptor excision circles (TRECs) through newborn screening for Severe Combined Immunodeficiency (SCID), which has been required in all 50 states in the USA since $2018[4,5]$. Congenital athymia is characterized by profound naïve T-cell deficiency with high susceptibility to infections, including opportunistic infections [6-8]. Patients with congenital athymia frequently develop manifestations of autologous graft-versus-host disease (GVHD) such as an eczematous rash, lymphadenopathy, and enteropathy. Autologous GVHD is similar to Omenn syndrome seen in SCID and is associated with oligoclonal T-cell expansion [8-10]. Patients with congenital athymia, oligoclonal T-cell expansion, and autologous GVHD are considered to have an atypical phenotype [11]. Complicating their care, patients also often present with clinical manifestations of associated genetic or syndromic conditions such as complete DiGeorge syndrome with or without 22q11.2 deletion, CHARGE syndrome (coloboma, heart defect, choanal atresia, growth or mental retardation, genital hypoplasia, and ear anomalies or deafness), forkhead box protein N1 (FOXN1) deficiency, and diabetic embryopathy [7, 12].

Historically, patients with congenital athymia typically die from infections or autoimmune sequelae within 2-3 years of life [13]. Current clinical management of congenital athymia consists of supportive care, which is geared toward minimizing risk of infection and treating infections and autologous GVHD $[9,14,15]$. Patients are recommended to be placed in immediate isolation following the diagnosis of congenital athymia (in line with procedures for primary immunodeficiencies) [14-17]. In the hospital, isolation usually requires contact and droplet precautions, infection prevention protocols for hospital staff, visitation restrictions, and, at times, even 
specialized air-flow rooms. At home, caregivers and family members are instructed to maintain strict isolation and hygiene procedures, as any interactions with individuals outside the immediate household can potentially expose the patient to pathogens that can lead to fatal infections, though isolation recommendations may vary by hospital or treating physician. As a result of these drastic requirements, profound immunodeficiency, and clinical sequelae of autologous GVHD, patients with congenital athymia can have prolonged inpatient stays in the hospital for weeks or months. In addition, patients receive multiple medications for prophylaxis and treatment of infections, immunosuppression for autologous GVHD, and frequent monitoring with medical tests to evaluate immune function $[9,13,15]$. Patients often require invasive procedures for their care, such as placement of a feeding tube or central line; central lines are frequently used in these patients to administer medications or draw blood [18]. Taken together, the high medical burden associated with supportive care for these patients has substantial clinical, emotional, social, and financial impact on caregivers and families.

There is a lack of published evidence characterizing the burden of congenital athymia on families and caregivers. As congenital athymia is reported in published literature primarily in the context of genetic and syndromic conditions $[7,12,19]$, there are few data characterizing the burden of congenital athymia independently. In fact, there is no published study that utilizes a validated patient-reported outcomes scale to characterize the specific impact of congenital athymia on the patient's health-related quality of life (HRQoL). Moreover, the burden of managing congenital athymia, including prolonged isolation and frequent healthcare utilization, on caregivers and families is unknown. Thus, this study sought to characterize the clinical, emotional, social, and financial burden of congenital athymia on pediatric patients who are receiving supportive care only as well as on their caregivers and families.

\section{METHODS}

This cross-sectional study utilized primary data research methods in adult caregivers of patients with congenital athymia to characterize the impact of congenital athymia. A web-based survey captured quantitative responses and was followed by a telephone-based interview led by an expert moderator who had a doctorate in clinical psychology. The study instrument included a screener, a web-enabled questionnaire, and a discussion guide designed to collect information about the holistic burden of illness across clinical, emotional, social, and financial domains. The study instrument was designed to differentiate the burden of congenital athymia from other comorbid conditions. To accomplish this differentiation between the clinical manifestations of congenital athymia from other comorbid conditions, a list was compiled of the clinical manifestations reported in congenital athymia in the literature and those associated with underlying comorbid conditions. The authors reviewed the list and, using their clinical experience treating patients with congenital athymia, assigned cause of clinical manifestations to either congenital athymia or to underlying syndromic comorbidities. In addition, caregivers were instructed to report the frequency of clinical manifestations associated with congenital athymia and the frequency of manifestations associated with underlying comorbid conditions. The discussion guide was designed to facilitate conversations in a semistructured manner to understand the areas of the disease that are most burdensome to the patients and families. Following the interviews, information was captured as transcribed notes in a response grid in Microsoft Excel ${ }^{\mathrm{TM}}$ (Microsoft Corporation, Redmond, WA, USA) to determine the most reported thematic elements of the interviews. Personal identifiable information was not collected.

Caregivers were recruited through online advocacy groups and social media support groups for families caring for patients with congenital athymia. To be considered eligible for the study, caregivers had to meet the following requirements: current caregivers had to 
be aged at least 18 years, have a caregiver-reported physician diagnosis of congenital athymia for the patient under their care, and be able to complete both the web-based survey and phone interview in English. Caregivers of the following patients were excluded from the study: deceased patients with congenital athymia and patients with hypothymia (low thymic function, but not absent). Only the primary caregiver for each patient was included in the study; in cases where there were coprimary caregivers, only one was included. All data on the impact of congenital athymia on patients, caregivers, and families were captured from the caregiver's perspective. Data were collected between November 2020 and January 2021 from respondents in the USA.

Caregivers of patients meeting the above criteria were included in the study. For patients currently receiving supportive care, all questions were framed for the past 12 months. For patients who had previously received investigational cultured human thymus tissue implantation, all questions were framed for the most recent 12 months they were receiving supportive care. To mitigate the potential recall bias for caregivers, a previously published selfreported confidence rating was used to assign a confidence score to each question for the respondents in this cohort [20]. Respondents were asked to rate how sure they were about the accuracy of their responses on a scale of $1=$ very unsure to 5 = very sure.

The questionnaire also included a validated patient-reported outcome scale-the parent proxy version of the Pediatric Quality of Life Inventory 4.0 Generic Core Scale (PedsQL) for ages 2-4 years [21]. The PedsQL consists of 23 items, which can be used to calculate a total score and component physical health summary score and psychosocial health summary score. The PedsQL ranges from 0 to 100, with a higher score representing a higher HRQoL. The scale is validated to be used with a 1-month recall and was only administered to caregivers of patients aged 2-4 years currently receiving supportive care.

Analyses were conducted using Q Research Software (Q Professional version 5.8.2.0, Displayr, Glebe, New South Wales, Australia) and
Microsoft Excel (Microsoft Corp.). Descriptive statistics were used to assess all quantitative data; medians with interquartile ranges (IQR) or means with standard deviations (SD) were derived for continuous variables and frequencies or percentages were derived for categorical variables, where appropriate. Data from qualitative interviews were systematically grouped into themes to identify similarities and dissimilarities between respondent experiences. Responses were grouped into tables, coded by 1 researcher, and cross-checked by another researcher. Subsequently, authors grouped these themes into categories and evaluated the most pertinent findings from the interviews.

\section{Compliance with Ethics Guidelines}

This study was reviewed by ADVARRA, a central Institutional Review Board (IRB, Identification Pro00047613). Following IRB review, this study was determined to have an exempt status (as defined by the US Department of Health and Human Services regulations found at 45 CFR 46.104[d][2]). This study was conducted in line with research ethics in accordance with the Helsinki Declaration of 1964 and its later amendments.

\section{RESULTS}

\section{Demographics and Characteristics}

Eighteen adult caregivers of patients who were currently or previously receiving supportive care for congenital athymia completed the quantitative survey and qualitative interview. Demographics and baseline characteristics of included patients and caregivers are summarized in Table 1.

All respondents identified as parental caregivers; half $(50 \%[n=9])$ were the coprimary caregivers with the patient's other parent (while the remaining 50\% identified as the sole "primary" caregiver). The median (IQR, Q1-Q3) respondent age at the time of participation was 39 (33-42) years. The caregivers were predominantly female $(89 \%[n=16]$ versus $11 \%[n=2])$. 
Table 1 Demographics and characteristics for patients with congenital athymia and their caregivers

$\begin{array}{lc}\text { Characteristics } & n=18\end{array}$

Patient sex, $n$ (\%)

Male

$10(56)$

Female

Caregiver sex, $n(\%)$

Male

Female

Patient experience with supportive care, $n(\%)$

Currently receiving supportive care

Previously received supportive care

Patient age in years, median (IQR Q1-Q3)

All patients

Currently receiving supportive care

$2(2-2)$

Previously received supportive care

$6(4-14)$

Caregiver age in years, median (IQR Q1-Q3)

$39(33-42)$

Time at which congenital athymia was diagnosed

At or around the time of birth

$11(61)$

At some point after birth

Age of diagnosis in months, median (IQR Q1-Q3)

$4(1-16)$

Caregiver's relationship with patient, $n$ (\%)

Parent

Geographic location, $n$ (\%)

Rural

Urban

Suburban

Time needed to reach medical center to receive congenital athymia-related care, $n$ (\%)

$<2 \mathrm{~h}$

$2-4 h$

4-6h

$2(11)$

$I Q R$ interquartile range, $Q$ quartile 
From a geographic perspective, most caregivers reported living in suburban areas (61\% $[n=11])$. Approximately one-quarter of caregivers $(28 \%[n=5])$ reported needing to drive $\geq 2 \mathrm{~h}$ to get to the hospital or medical center that provides care for the patient. Four caregivers reported needing to either permanently move to be closer to their healthcare center or be unable to move because of current proximity to their healthcare center. In addition, eight caregivers reported needing to temporarily relocate closer to a treatment center.

More than half $(61 \%[n=11])$ of patients were diagnosed with congenital athymia at or around the time of their birth, and the remainder were diagnosed after birth (39\% $[n=7]$ ), at a median (IQR, Q1-Q3) age of 4 (1-16) months. The sample consisted of a similar percentage of male $(56 \%[n=10])$ and female $(44 \%[n=8])$ patients. The median (IQR, Q1-Q3) patient age at the time of participation was 5 years ( $2-13$ years). For patients who were currently receiving supportive care $(n=5)$, the median (IQR, Q1-Q3) age at time of participation in this study was $2(2-2)$ years. For patients who had received supportive care in the past $(n=13)$, the median (IQR, Q1-Q3) age at the time of participation in this study was 6 (4-14) years. Across all questions in the quantitative survey, caregivers reported an average self-rated confidence score of 4.6, indicating very high confidence between "quite sure" and "very sure;" the mean self-rated confidence score did not go below 3.9 for any question.

\section{Overall Burden}

Caregivers reported the top three ways that congenital athymia impacted the patient and the family (Fig. 1). Answers included the following: living in isolation and lack of normalcy for patients $(100 \%[n=18])$; caregiver emotional burden such as fear of death, infections, and worries about the future $(100 \%[n=18])$; financial hardship due to loss of family income $(78 \%[n=14])$; being unable to meet family or friends $(72 \%[n=13])$; burden of medical care on the patient $(67 \%[n=12])$; lack of normalcy for sibling or family $(67 \%[n=12])$; and impact on patient's physical, mental, and social development $(61 \%[n=11])$. Caregivers also reported impact on sibling's development $(50 \%[n=9])$, lack of resources about congenital athymia or lack of support for families $(17 \%[n=3])$, needing to move or travel for treatment $(17 \%$ $[n=3])$, and patients only meeting people wearing personal protective equipment $(17 \%$ $[n=3])$.

\section{Disease Burden}

Patients experienced frequent life-threatening clinical manifestations associated with congenital athymia. Notably, nearly a quarter of patients experienced sepsis $(22 \%[n=4])$ at a frequency of every 3-4 months. Caregivers were asked about otolaryngologic, genitourinary, skin, gastrointestinal, and lung disorders, including infections in these organs. Caregivers reported that more than half the patients experienced endocrine system disorders (61\% $[n=11])$ and otolaryngologic disorders (61\% $[n=11] ; \quad$ Fig. 2a). Patients also experienced genitourinary disorders $(44 \%[n=8])$, skin disorders $(39 \%[n=7])$, gastrointestinal disorders $(33 \%[n=6])$, kidney injury or damage due to medication $(33 \%[n=6])$, and lung disorders $(22 \%[n=4])$. Several of the clinical manifestations were described as severe, including lung, skin, and gastrointestinal disorders; kidney injury or damage due to medications; and endocrine system disorders. Nearly all clinical manifestations were reported to occur as both chronic and acute manifestations in patients, except gastrointestinal disorders that were all considered to be chronic.

Clinical manifestations of congenital athymia occurred with high frequency. One-third of patients $(33 \%[n=6])$ experienced $>4$ unique types of clinical manifestations of congenital athymia and more than half of patients $(67 \%$ [ $n=12]$ ) experienced between 1 and 4 unique types of clinical manifestations of congenital athymia in the most recent 12 months while they were receiving supportive care (Fig. 2b). Among reported clinical manifestations considered to be related to congenital athymia, otolaryngologic and gastrointestinal disorders 


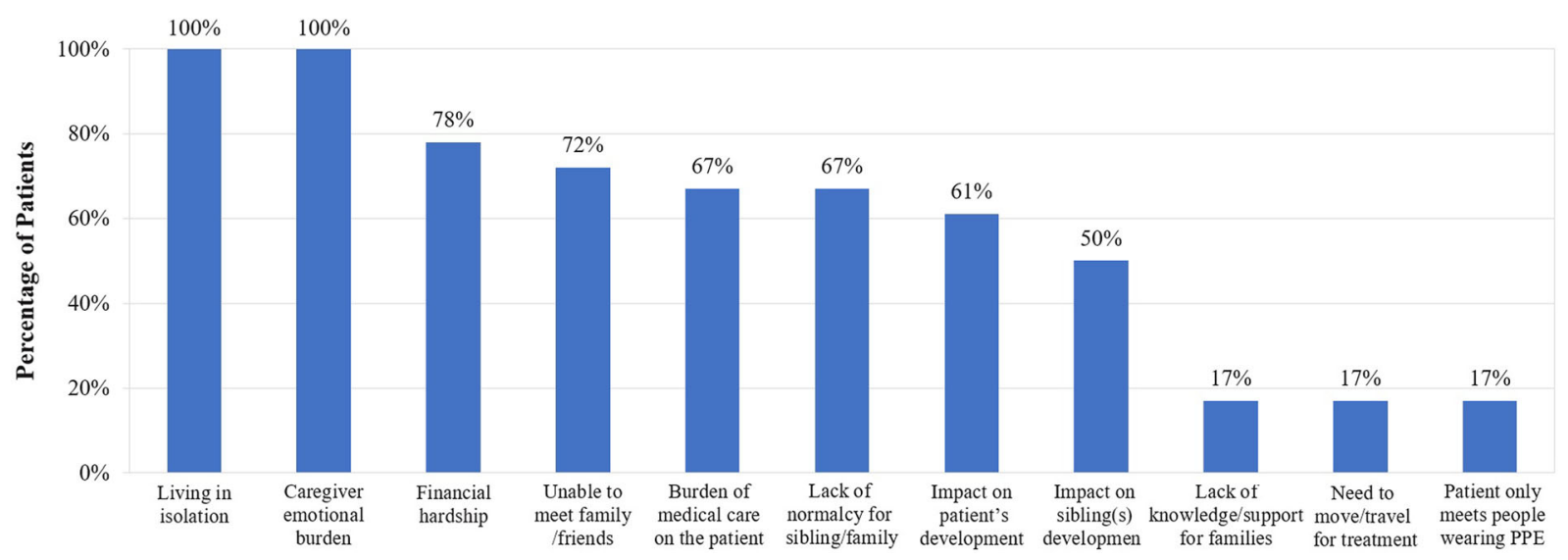

Fig. 1 Top three ways congenital athymia impacted patients, caregivers, and families. Caregivers were asked to report the top three most important ways in which having congenital athymia has impacted their loved one's

commonly occurred more than once a month. Manifestations considered to be related to congenital athymia most commonly occurring every 3-4 months included endocrine system and genitourinary disorders. Many different specialists were reported as part of patients' care teams, including pediatricians $(94 \%[n=17])$, immunologists $(89 \%[n=16])$, infectious disease specialists $(28 \%[n=5])$, hematologists $(17 \%[n=3])$, or other specialties $(44 \%[n=8]$; Table 2). Immunologists were the commonly reported primary care physicians $(78 \%[n=14])$.

Nearly all the patients (94\%) experienced at least one comorbid condition not directly associated with their congenital athymia (Table 3). In addition to clinical manifestations associated with their congenital athymia, patients experienced feeding or growth concerns $(83 \%[n=15])$, congenital heart defects $(67 \%[n=12])$, hypocalcemia $(50 \%[n=9])$, respiratory conditions $(44 \% \quad[n=8])$, hypoparathyroidism $(22 \%[n=4])$, and other comorbidities $(22 \%[n=4])$. One patient caregiver reported no comorbid conditions.

Caregivers reported that within a 12-month period, nearly all patients $(94 \%[n=17])$ required at least one hospitalization (Table 4). Most patients $(78 \%[n=14])$ were in the hospital for at least 1 month, and nearly a quarter $(22 \%[n=4])$ were in the hospital for > 9 months. Most of the hospitalized patients life as well as their own life/family's life. Key themes were distilled from responses and are presented by total number of respondents who mentioned the item as one of their top three

required specialized care in the pediatric intensive care unit during hospitalization $(65 \%$ $[n=11]$ of patients who were hospitalized $[n=17])$. Caregivers needing to stay in the room full-time with the patient during their inpatient hospitalization reported that no additional people outside the patient's doctors or nurses could enter. Several caregivers specifically described the room the patient stayed in as an "isolation room."

Patients with congenital athymia had high utilization of prescription medications and nonprescription medications in the most recent 12 months while receiving supportive care (Table 5). Prescription medication use included immunoglobulin replacement therapy (94\% $[n=17]) ;$ antibiotic, antiviral, or antifungal medications for prophylaxis (94\% $[n=17])$ and treatment $(72 \% \quad[n=13])$; and immunosuppressant medications $(39 \%[n=7])$. Nonprescription medication use (e.g., acetaminophen, ibuprofen, antacids, antihistamines) was also commonly reported $(89 \%[n=16])$.

Patients also utilized high rates of surgical or diagnostic procedures associated with their congenital athymia; nearly three-quarters of patients $(72 \%[n=13])$ received $\geq 5$ unique types of procedures in the most recent 12 months while receiving supportive care (Fig. 3a). Among these, radiology scans (94\% $[n=17])$, sedation $(89 \%[n=16])$, feeding tube 

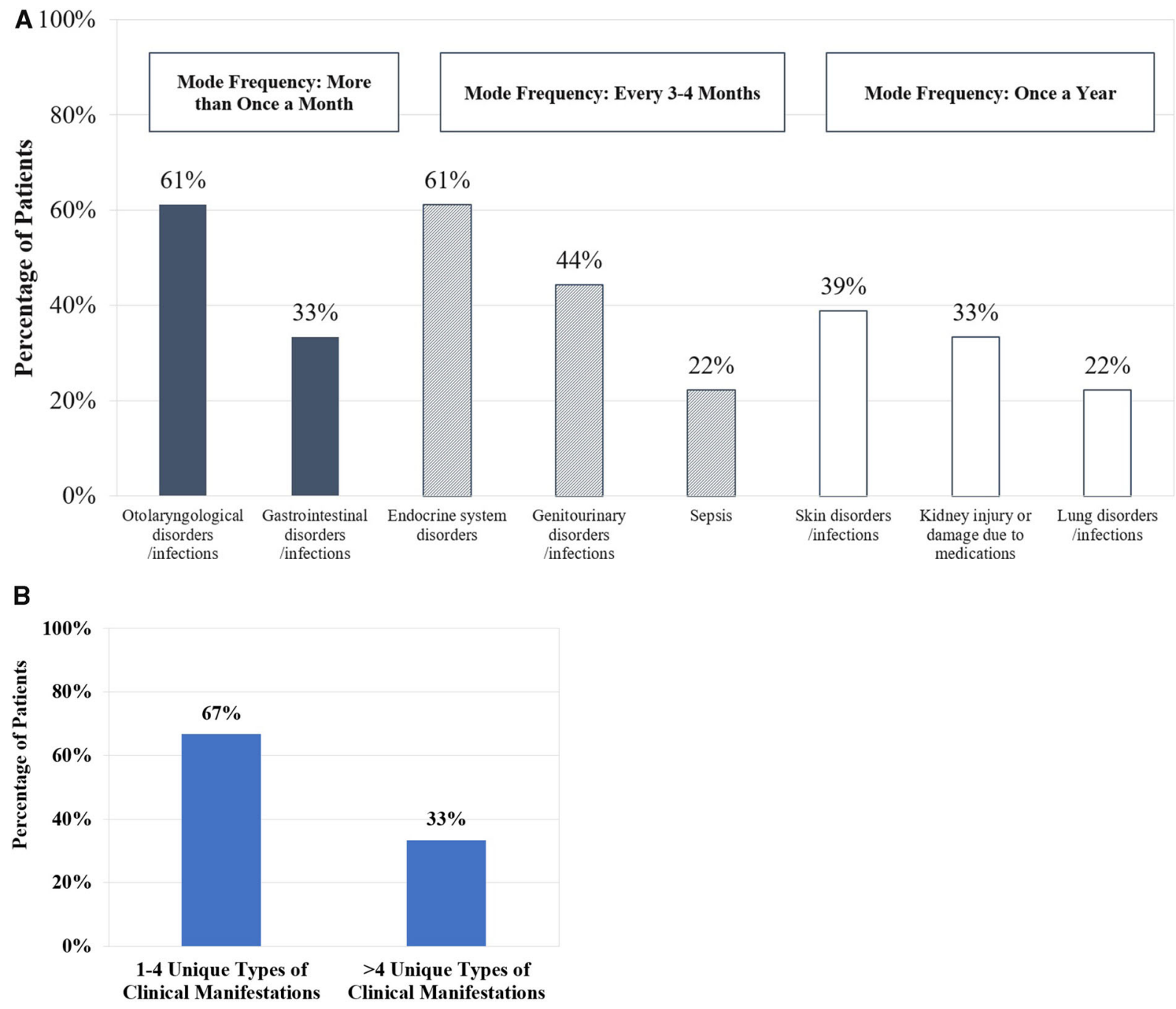

Fig. 2 Frequency of clinical manifestations of congenital athymia in the most recent 12 months while receiving supportive care. a Experience with and frequency of clinical manifestations of congenital athymia among patients in the most recent 12 months while receiving supportive care. Clinical manifestations occurred at a mode

placement $(72 \%[n=13])$, central line placement $(67 \%[n=12])$, peripherally inserted central catheters $(61 \%[n=11])$, and bronchoscopy (56\% $[n=10])$ were reported in more than half of patients (Fig. 3b). Caregivers characterized the frequent medical procedures, including blood tests, central line placement, sedation, and feeding tubes, as repeated trauma for patients. frequency of more than once a month (filled bars), every 3-4 months (hatched bars), or once a year (open bars). b Percentage of patients who experienced one or more types of clinical manifestations of congenital athymia in the most recent 12 months while receiving supportive care

Caregivers communicated the burden of around-the-clock medications and the frequent procedures required for patients. Illustrating this burden, caregivers described their homes as looking like a hospital because of the amount of medical equipment and supplies needed. Four caregivers also noted feeling reluctant or worried about utilizing nursing and home healthcare services because of the potential exposure to infection. Half of caregivers $(50 \%[n=9])$ 
Table 2 Healthcare professionals reported for patient care and management for congenital athymia

\begin{tabular}{lr}
\hline & $\boldsymbol{n}=\mathbf{1 8}$ \\
\hline Patient's disease management and care team, $n(\%)$ \\
Pediatrician & $17(94)$ \\
Immunologist & $16(89)$ \\
Infectious disease specialist & $5(28)$ \\
Hematologist & $3(17)$ \\
Other & $8(44)$ \\
Patient's primary physician, $n(\%)$ & \\
Immunologist & $14(78)$ \\
Hematologist & $2(11)$ \\
Pediatrician & $2(11)$ \\
\hline
\end{tabular}

Other includes endocrinologist; ear, nose, and throat specialist; ophthalmologist; cardiologist; nephrologist; neuropsychologist; urologist; audiologist; and psychiatrist

Table 3 Frequency of comorbidities in patients with congenital athymia in the most recent 12 months while receiving supportive care

$n=18$

Comorbidities, $n$ (\%)

Feeding or growth concerns

Congenital heart defects

Hypocalcemia

Respiratory conditions

Hypoparathyroidism

Other

None

reported utilizing home healthcare services in the most recent 12 months while the patient was receiving supportive care (Table 5); these services were used typically at a frequency of once per week.

Caregivers emphasized the impact of isolation on the patient and family and the constant 
Table 5 Medication, supply, and home healthcare utilization in patients with congenital athymia in the most recent 12 months while receiving supportive care

\begin{tabular}{lr}
\hline & $n=\mathbf{1 8}$ \\
\hline Medication use to prevent or treat clinical manifestations, $n$ (\%) & \\
Prescription medication & $17(94)$ \\
Immunoglobulin replacement medications & $17(94)$ \\
Prophylactic antibiotics, antivirals, or anti-infectives & $13(72)$ \\
Antibiotics, antivirals, or anti-infectives to treat infections & $7(39)$ \\
Immunosuppressant medications & $16(89)$ \\
Non-prescription medication & $18(100)$ \\
Supplies to prevent or treat clinical manifestations, $n(\%)$ & $17(94)$ \\
Home medical supplies & $9(50)$ \\
Non-medical supplies & \\
Home healthcare, $n(\%)$ &
\end{tabular}

fear of infections. Isolation, as reported by caregivers, limited the patient's ability to meet with extended family or even siblings for long periods. Caregivers reported that the isolation required for infection control led to missed social opportunities and interactions affecting patients' emotional and social development. Caregivers also communicated the impact of isolation on their own lives, including the feeling of separation from their support system of family and friends. They reported loss of normalcy in their lives, including activities such as going to the grocery store or out to dinner. The significant consequence of having a patient with congenital athymia for the family extends to siblings' lives as well. Caregivers reported having to remove siblings from school and/or restrict their ability to interact with peers or participate in school activities to minimize infection risk.

All caregivers $(100 \% \quad[n=18])$ reported needing home medical supplies to care for patients with congenital athymia (e.g., gloves, masks, syringes), and nearly all (94\% $[n=17])$ reported needing nonmedical supplies (e.g., high-efficiency particulate air purifiers or filters, hand sanitizers, home cleaning supplies; Table 5).

\section{Quality of Life Burden}

Four patients met the criteria for administration of parent proxy version of the PedsQL (i.e., those patients currently receiving supportive care aged between 2 and 4 years). One patient currently receiving supportive care was aged $<2$ years and therefore did not qualify for the assessment. Patients with congenital athymia had a mean (SD) total score of 56.60 (27.9), with a component physical summary score of 53.91 (29.3) and psychosocial summary score of 58.75 (27.5; Fig. 4).

Caregivers reported the impact of congenital athymia on their ability to perform specific tasks, using a scale of $1-7$, with 7 equating to "very high impact" (Fig. 5). All dimensions were rated with a mean score $>6$. Caregivers reported high mean impact on their ability to make plans for the future (7.0), socialize with friends and family (6.78), travel for pleasure (6.72), get a good night's sleep (6.56), and conduct normal activities of daily living (6.27).

Caregivers communicated the emotional toll of caring for a patient with congenital athymia. They described a constant fear that their child would die, feeling like they were responsible for 

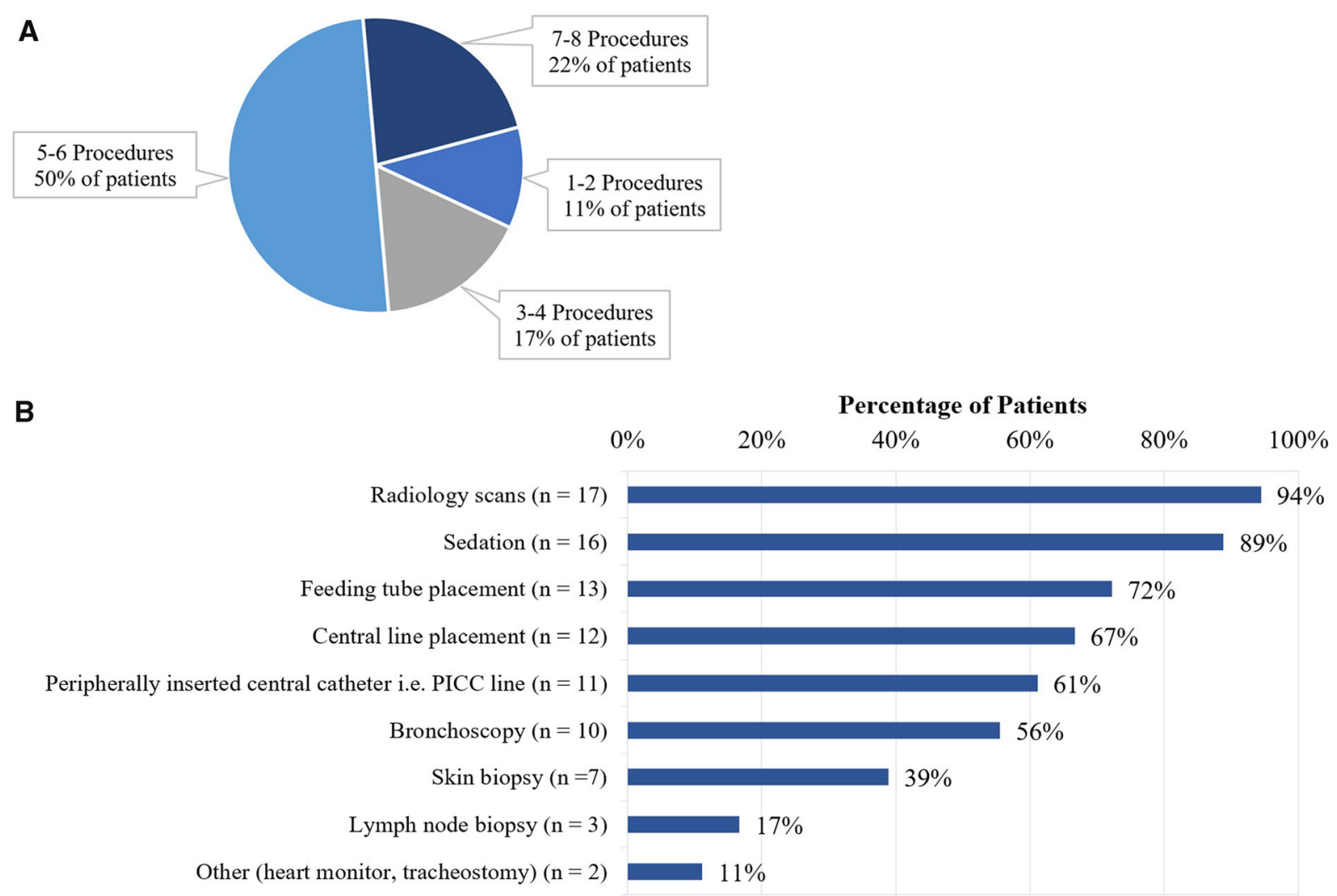

Fig. 3 Surgical and diagnostic procedure utilization in patients with congenital athymia in the most recent 12 months while receiving supportive care. a Percentage of patients who underwent one or more unique types of surgical or diagnostic procedures associated with congenital

keeping their child healthy and free of infection. Caregivers frequently used words like overwhelmed, scared, exhausted, and anxious to describe the moment that their child was diagnosed with congenital athymia.

\section{DISCUSSION}

To our knowledge, this is the first study evaluating the clinical, emotional, social, and financial burden of congenital athymia on patients, caregivers, and their families. To date, the existing literature on congenital athymia has been restricted to characterizing the clinical manifestations of the condition, largely in the context of other associated genetic and syndromic conditions $[7,12,19]$. As a result, there athymia in the most recent 12 months while receiving supportive care. b Percentage of patients utilizing specific surgical and diagnostic procedures in the most recent 12 months while receiving supportive care

is no reliable published information on incidence and prevalence of congenital athymia. Between 2017 and 2020, 71 patients were diagnosed and referred to Duke University for investigational cultured human thymus tissue implantation (on average, about 18 patients annually). The inclusion criteria for entry into the clinical trial of investigational cultured human thymus tissue implantation at Duke University are athymia with circulating $\mathrm{CD}^{+}$ $\mathrm{CD}_{45 \mathrm{RA}^{+}} \mathrm{CD} 62 \mathrm{~L}^{+} \mathrm{T}$ cells of $<50 / \mathrm{mm}^{3}$ or $<5 \%$ of the total T-cell count on two separate flow cytometry analyses, one performed within 3 months and one within 1 month prior to implantation [22]. The number of patients referred to Duke captures most cases of congenital athymia, as investigational treatment at Duke is the only current option for these 


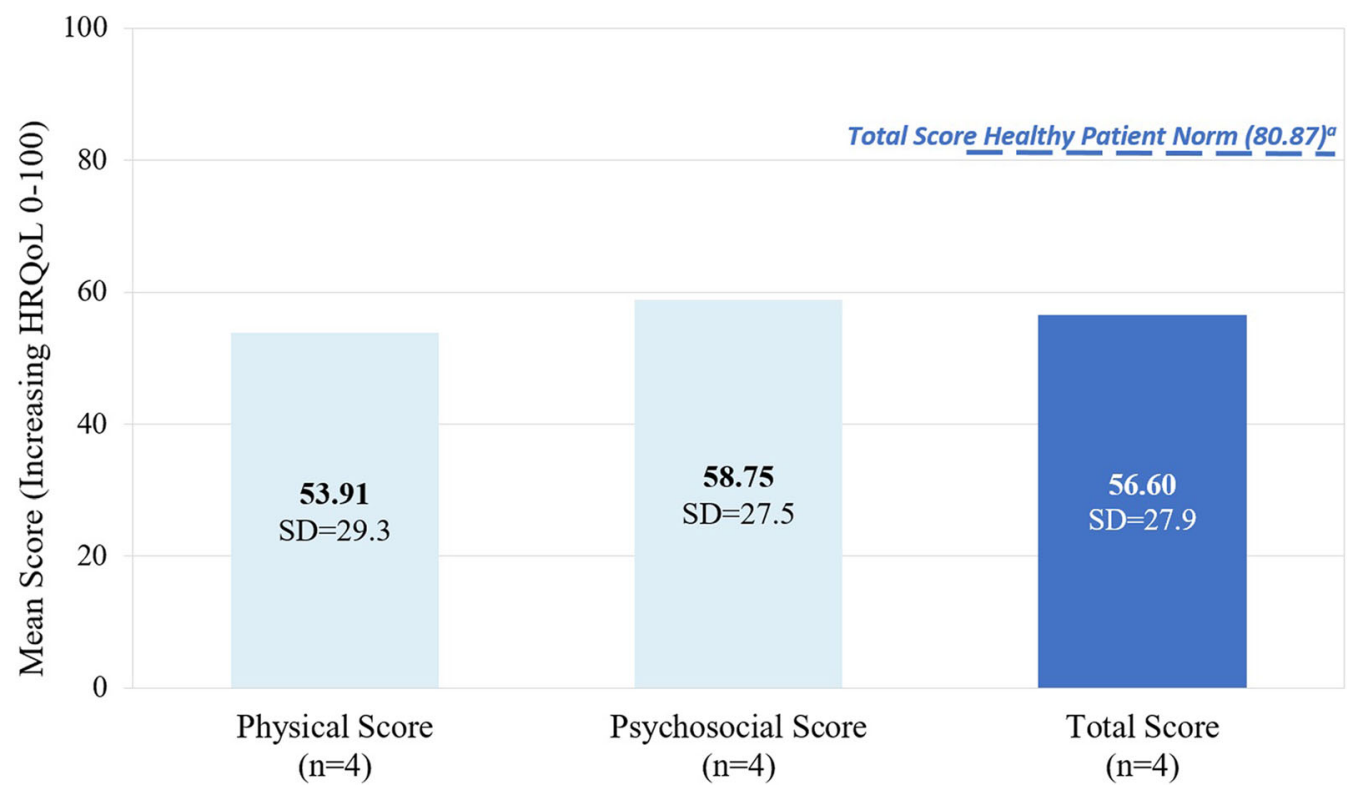

Fig. $\overline{4}$ Pediatric Quality of Life Inventory (PedsQL) 4.0 Generic Core Scale Scores in patients with congenital athymia. The parent proxy of the PedsQL 4.0 Generic Core Scale was administered to caregivers of patients with congenital athymia aged 2-4 years who were currently receiving supportive care. ${ }^{\text {a }}$ The dark blue dashed line represents the reference value for the published total score in healthy pediatric patients [21]

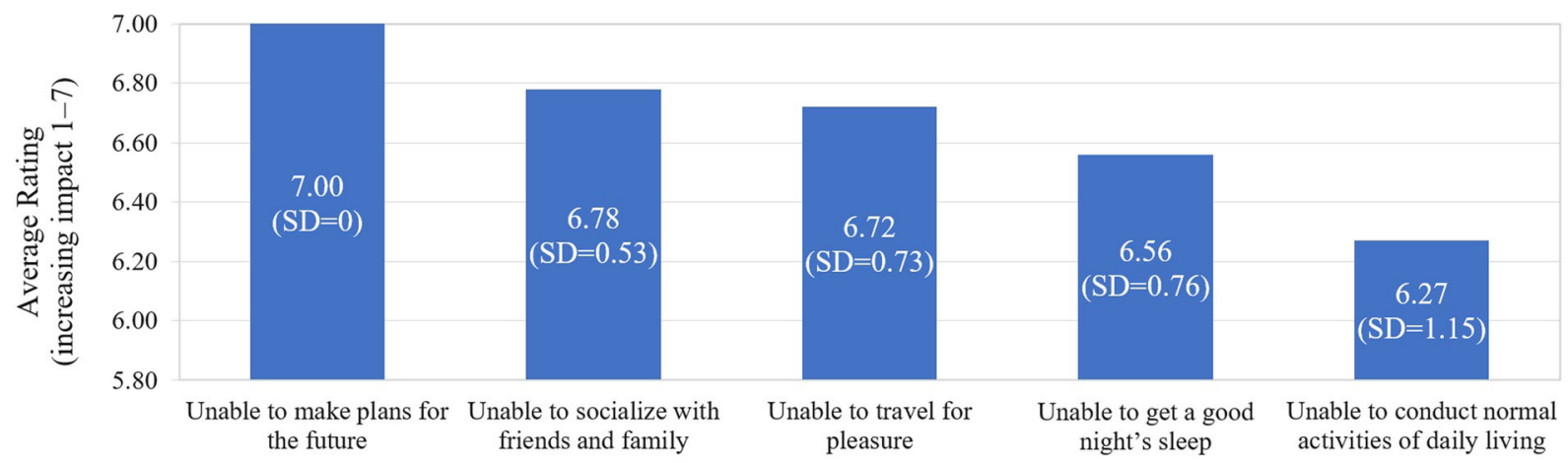

Fig. 5 Impact of congenital athymia on caregivers' lives across multiple dimensions. Caregivers were asked to rate the impact of their loved one's congenital athymia on their ability to perform specific tasks. Ratings are on a scale of 1

patients in the US. Thus, the sample size in this study $(n=18)$ is reasonable, as it is similar to the number of patients referred to Duke annually and a fair estimation for the annual incidence of the disorder in the US.

Although several published studies report the occurrence of clinical manifestations in patients with congenital athymia [3, 6, 8, 23], to 7 where $1=$ "no impact" and $7=$ "very high impact." Scores are reported as the average rating across all caregivers $(n=18)$

the data are not always captured systematically across patients, and this information is frequently described only in case reports [7, 9]. We set out to characterize the volume of clinical burden, considering both the number of patients experiencing each type of clinical manifestation and the frequency with which they occur, in patients with congenital athymia 
over a 12-month period. Overall, most patients experienced numerous and frequent clinical manifestations associated with congenital athymia across multiple organs, including otolaryngologic, skin, lung, gastrointestinal, and genitourinary disorders or infections as well as endocrine system disorders. A notable finding was that nearly a quarter of patients with congenital athymia experienced sepsis every 3-4 months.

As a result of the strict isolation required for patients with congenital athymia [14-17], patients are unable to physically explore the world beyond their homes or socialize and bond with extended family or peers, potentially causing patients to miss critical milestones. In fact, several caregivers reported concern that their child had already experienced or would experience delays in psychosocial and emotional development associated with their sustained isolation. The unique contribution of prolonged isolation on development is difficult to differentiate from cognitive delays or intellectual disability associated with syndromic comorbidities, such as 22q11.2 deletion syndrome. However, data demonstrating the value of social interaction in infancy and childhood [24] suggest it is possible that these patients experience long-term effects of early isolation.

Remarkably, $100 \%$ of caregivers reported the need to live in isolation and caregiver emotional burden as the top ways that congenital athymia affected them and their families. Given that patients with congenital athymia have historically died within the first 2-3 years of life with only supportive care, parents of these patients expressed considerable fear that their child would die and an overwhelming feeling of responsibility for keeping their child safe. This study revealed that these feelings were compounded by isolation from support systems of extended family and friends, with caregivers seemingly having to navigate many experiences alone and mourn the loss of normalcy in their lives. Moreover, social isolation and loneliness have been shown to be associated with depression and impaired cognitive health, and potentially an increased economic burden [25-27]. Older siblings were also impacted, as siblings were frequently withdrawn from school and homeschooled following the patient's diagnosis, causing siblings to miss out on critical social interactions and possibly learning progression. In addition, caregivers voiced concerns about siblings needing to maintain isolation to minimize infection risk for the patient. Caregivers worry that the inability of siblings to freely interact with families and friends outside their household or participate in team sports would negatively impact their social development. Collins et al. recently published recommendations for supportive care of patients with congenital athymia [28], but no national or international consensus guidelines exist for managing patients with congenital athymia. Consensus guidelines would ensure that isolation procedures are adequate for minimizing infection risk without unnecessarily increasing the considerable burden of isolation on patients and their families.

Due to the extensive clinical burden, patients with congenital athymia experience high healthcare resource utilization, primarily in the form of lengthy inpatient hospital stays in specialty units such as pediatric intensive care units and multiple medical procedures. Patients with congenital athymia also required multiple medications to minimize their risk of infection and manage manifestations of autologous GVHD. These medications, primarily antimicrobials and immunosuppressants, can lead to adverse reactions as evidenced by the $33 \%$ of patients who developed kidney damage due to frequent and long-term use of nephrotoxic medications. The prophylactic antibiotics, antivirals, or antifungal medications that were utilized by nearly all patients have the potential to predispose patients to antimicrobial-resistant infections. Mohammadinejad et al. [29] found that antibiotic resistance appears to be higher among patients with primary immunodeficiencies who receive significant antibiotic prophylaxis and treatment compared with immunocompetent patients.

Caregivers also reported multiple indirect costs associated with managing their child's congenital athymia. Nearly all caregivers reported one parent needing to give up or take time off from their work to take care of their child full time, resulting in loss of income. Only 
half of caregivers reported utilizing home healthcare services. Caregivers reported that the fear of exposing their child to infection kept them from getting much needed nursing help, requiring caregivers to spend significant time providing direct medical care to patients and traveling to doctors' appointments. Nearly onethird of caregivers reported needing to drive $\geq 2 \mathrm{~h}$ to receive care at a medical center; likely this was due to the patient's need to receive care at a tertiary medical center. Several caregivers also reported having to permanently move to be in closer proximity to a medical center. Frequent hospital visits and complex medical care associated with this condition also impacted the primary income-earner's career because of absences from work to address medical issues or because of a lack of productivity while working due to concerns for their child's health. Caregivers reported giving up desirable career choices for fear of negative impact on their sick child (such as declining a career move to avoid losing current health insurance benefits), and educational sacrifices were noted across the family members. Caregivers reported changing or delaying plans to pursue education that would benefit their career and earning potential to care for the patient.

As measured by the validated PedsQL scale, patients with congenital athymia had a substantially lower mean total score compared with published literature of healthy pediatric patients (56.60 versus 80.87) [21]; the PedsQL takes into account patients' overall HRQoL, not the experience specific to their congenital athymia. The total score in patients with congenital athymia was also lower compared with other conditions, such as pediatric cancer (69.70) and end-stage renal disease (67.02) $[30,31]$. To contextualize the impact of congenital athymia on HRQoL, scores also were compared with similarly debilitating congenital conditions with substantial burden for patients and families. The total score of patients with congenital athymia was similar to that of patients with spinal muscular atrophy (53.35) and 22q11.2 deletion syndrome (50.98) on the PedsQL parent proxy report [32, 33]. To the authors' knowledge, this is the first study evaluating quality of life in patients with congenital athymia.

There are several limitations that should be considered while reviewing this study. The first is the extremely rare nature of congenital athymia and limited sample size of the evaluated cohort. Given that there are 17 to 24 incident cases in the US annually, the sample size of 18 patients appears appropriate. To help account for the more limited sample size, the authors structured the study methods to be able to account for a breadth of potential experiences by utilizing qualitative interviewing, which introduces another limitation, as not all results could be quantified. In addition, only four patient caregivers were eligible to complete the PedsQL. Because the PedsQL is only validated in patients aged 2 years and older for a 1 -month recall period, the sample was limited to caregivers of patients ( $\geq 2$ years old) that were currently receiving supportive care $(n=4)$. However, the sample size appears reasonable given the low estimated prevalence of congenital athymia. Another limitation of the study is that responses of certain caregivers may be subject to recall bias as respondents may not be able to remember previous events or experiences, omit details, or experience memories that are influenced by subsequent events and experiences. In the study, a potential for recall bias was present for caregivers of patients who had previously received supportive care $(n=13)$ and were asked to recall the 12 months when they were last receiving supportive care. To help mitigate this bias, these caregivers were asked to rate, for each question, their assessment of the accuracy of their response on a 1-5 scale (5 being the highest confidence). Additionally, some questions were asked with categorical response options instead of continuous response options for this cohort.

Another potential limitation is that certain clinical manifestations associated with congenital athymia in these patients may have overlapping features of underlying syndromic comorbidities. For example, patients may have chronic sinus/ear, nose, and throat problems due to an anatomical abnormality or due to a chronic respiratory viral infection. In this study, care was taken to distinguish the clinical 
manifestations associated with congenital athymia from those stemming from other genetic malformations, including getting consensus from expert advisors on the list of clinical manifestations associated with congenital athymia versus comorbid conditions included in the survey and instructing caregivers to report clinical manifestations associated with congenital athymia separately from other comorbid conditions. Although we did not ask caregivers to provide patients' associated genetic or syndromic conditions, congenital athymia can be associated with FOXN1 deficiency, complete DiGeorge syndrome with or without 22q11.2 deletion, CHARGE syndrome, and diabetic embryopathy [28].

In our study, 13 patients had previously received investigational cultured human thymus tissue implantation. Although our study was not designed to evaluate changes to disease burden or quality of life post-implantation, patients treated with investigational cultured human thymus tissue implantation have been shown to have a significant decrease in the number of infections 6 to $\leq 12$ months versus the first 6 months post-implantation $(p<0.001)$, with a Kaplan-Meier estimated survival rate of $76 \% 2$ years post-implantation [22]. Future research is warranted to evaluate the change in disease burden and quality of life in patients with congenital athymia and their families post-investigational cultured human thymus tissue implantation.

A final consideration for the data is that caregivers included in this study were recruited through advocacy/support groups for congenital athymia and thus likely had more access to resources and support, which may have biased their responses compared with other caregivers' responses. Moreover, although only caregivers of living patients were included, patients with congenital athymia typically die within the first 3 years of life with only supportive care. Taken together, it is possible that results of this study may underestimate the full burden of congenital athymia across all families. Therefore, in the future, areas to consider exploring include the long-term impact (i.e., $>12$ months) of congenital athymia on the patient and caregiver's lives, social development, potential post- traumatic stress, or other elements of psychosocial development.

\section{CONCLUSIONS}

This study provides the most comprehensive picture of the burden of congenital athymia thus far presented in the literature. This study demonstrated the clinical, emotional, social, and financial impact of congenital athymia on patients, caregivers, and families. Both the quantitative survey and qualitative interviews revealed significant impacts of congenital athymia, including social isolation, caregiver and family emotional burden, financial hardship, undesirable career and educational choices, and negative impact on siblings. Future research should focus on improving the care of patients with congenital athymia to reduce morbidity and mortality of these patients and minimize the burden of care.

\section{ACKNOWLEDGEMENTS}

We thank Megan Cooper, MD, PhD (Associate Professor, Rheumatology/Immunology, Department of Pediatrics, Washington University School of Medicine), for her expert advice on the therapeutic area and her contribution to study design. We also thank Bhagyashree Oak, PhD (Trinity Life Sciences), for her contribution to study design and data analysis, and Lisa Bailey, PhD (Trinity Life Sciences), for serving as the expert moderator during cognitive interviews. Megan Cooper, Bhagyashree Oak, and Lisa Bailey received consulting fees from Enzyvant Therapeutics, Inc. We thank the participants of the study.

Funding. This study was funded by Enzyvant Therapeutics, Inc. Enzyvant Therapeutics, Inc., was involved in study design; the collection, analysis, and interpretation of data; writing of the report; and the decision to submit the paper for publication. Enzyvant Therapeutics Inc. is funding the Advances in Therapy Rapid Service and Open Access Fees. 
Medical Writing and Editorial Assistance. Medical writing assistance was provided by Emily Sharpe, PhD, of Trinity Life Sciences. Emily Sharpe received consulting fees from Enzyvant Therapeutics, Inc. Editorial assistance, funded by Enzyvant Therapeutics, Inc., was provided by Lisa M Pitchford, PhD, of JB Ashtin.

Authorship. All named authors meet the International Committee of Medical Journal Editors (ICMJE) criteria for authorship for this article, take responsibility for the integrity of the work as a whole, and have given their approval for this version to be published.

Author Contributions. Conceptualization: Elena Hsieh, Sarah Kulke, Abigail Silber, Matthew O'Hara, and Cathleen Collins; Methodology: Elena Hsieh, Sarah Kulke, Abigail Silber, Matthew O'Hara, and Cathleen Collins; Investigation: Elena Hsieh, Sarah Kulke, Abigail Silber, Matthew O'Hara, and Cathleen Collins; Data curation and analysis: Elena Hsieh, Julie Kim-Chang, Sarah Kulke, Abigail Silber̃, Matthew O'Hara, and Cathleen Collins; Writing-original draft preparation: Sarah Kulke and Abigail Silber; Writing-review and editing: Elena Hsieh, Julie Kim-Chang, Matthe $\bar{w} \mathrm{O}^{\prime}$ Hara, and Cathleen Collins; Supervision: Elena Hsieh, Sarah Kulke, Abigail Silber, Matthew O'Hara, and Cathleen Collins. All authors read and approved the final manuscript.

Prior Presentation. A subset of the data included in this manuscript was presented as a poster presentation at the Clinical Immunology Society 2021 Virtual Annual Meeting, April 14-17, 2021.

Disclosures. Julie J. Kim-Chang has nothing to disclose. Elena Hsieh, Cathleen Collins, Abigail Silber, and Matthew O'Hara received consulting fees from Enzyvant Therapeutics, Inc. Sarah Kulke is the vice president of Medical Affairs at Enzyvant Therapeutics, Inc. (since May 2020) and, therefore, receives a salary, annual performance bonus, and cash based on a long-term incentive program. Enzyvant Therapeutics Inc., was involved in study design; the collection, analysis, and interpretation of data; the writing of the report; and the decision to submit the paper for publication.

Compliance with Ethics Guidelines. This study was reviewed by ADVARRA, a central Institutional Review Board (IRB, Identification Pro00047613). Following IRB review, this study was determined to have an exempt status (as defined by the US Department of Health and Human Services regulations found at 45 CFR 46.104[d][2]). This study was conducted in line with research ethics in accordance with the Helsinki Declaration of 1964 and its later amendments.

Data Availability. The datasets generated during and/or analyzed during the current study are available from the corresponding author on reasonable request.

Open Access. This article is licensed under a Creative Commons Attribution-NonCommercial 4.0 International License, which permits any non-commercial use, sharing, adaptation, distribution and reproduction in any medium or format, as long as you give appropriate credit to the original author(s) and the source, provide a link to the Creative Commons licence, and indicate if changes were made. The images or other third party material in this article are included in the article's Creative Commons licence, unless indicated otherwise in a credit line to the material. If material is not included in the article's Creative Commons licence and your intended use is not permitted by statutory regulation or exceeds the permitted use, you will need to obtain permission directly from the copyright holder. To view a copy of this licence, visit http://creativecommons.org/licenses/by$\mathrm{nc} / 4.0 /$.

\section{REFERENCES}

1. Regulation (EU) No 536/2014 of the European parliament and of the council of 16 April 2014 on clinical trials on medicinal products for human use, and repealing Directive 2001/20/EC (2014). 
2. Gordon J, Manley NR. Mechanisms of thymus organogenesis and morphogenesis. Development. 2011;138(18):3865-78.

3. Markert ML, Sarzotti M, Ozaki DA, Sempowski GD, Rhein ME, Hale LP, et al. Thymus transplantation in complete DiGeorge syndrome: immunologic and safety evaluations in 12 patients. Blood. 2003;102(3):1121-30.

4. Amatuni GS, Currier RJ, Church JA, Bishop T, Grimbacher E, Nguyen AA, et al. Newborn screening for severe combined immunodeficiency and T-cell lymphopenia in California, 2010-2017. Pediatrics. 2019;143(2):e20182300.

5. Kwan A, Abraham RS, Currier R, Brower A, Andruszewski K, Abbott JK, et al. Newborn screening for severe combined immunodeficiency in 11 screening programs in the United States. JAMA. 2014;312(7):729-38.

6. Janda A, Sedlacek P, Honig M, Friedrich W, Champagne M, Matsumoto T, et al. Multicenter survey on the outcome of transplantation of hematopoietic cells in patients with the complete form of DiGeorge anomaly. Blood. 2010;116(13):2229-36.

7. Markert ML, Marques JG, Neven B, Devlin BH, McCarthy EA, Chinn IK, et al. First use of thymus transplantation therapy for FOXN1 deficiency (nude/SCID): a report of 2 cases. Blood. 2011;117(2):688-96.

8. Davies EG, Cheung M, Gilmour K, Maimaris J, Curry J, Furmanski A, et al. Thymus transplantation for complete DiGeorge syndrome: European experience. J Allergy Clin Immunol. 2017;140(6):166070 e16.

9. Markert ML, Alexieff MJ, Li J, Sarzotti M, Ozaki DA, Devlin $\mathrm{BH}$, et al. Complete DiGeorge syndrome: development of rash, lymphadenopathy, and oligoclonal T cells in 5 cases. J Allergy Clin Immunol. $2004 ; 113(4): 734-41$.

10. Selim MA, Markert ML, Burchette JL, Herman CM, Turner JW. The cutaneous manifestations of atypical complete DiGeorge syndrome: a histopathologic and immunohistochemical study. J Cutan Pathol. 2008;35(4):380-5.

11. Villa A, Notarangelo LD, Roifman CM. Omenn syndrome: inflammation in leaky severe combined immunodeficiency. J Allergy Clin Immunol. 2008;122(6):1082-6.

12. Markert ML, Devlin BH, Alexieff MJ, Li J, McCarthy EA, Gupton SE, et al. Review of 54 patients with complete DiGeorge anomaly enrolled in protocols for thymus transplantation: outcome of 44 consecutive transplants. Blood. 2007;109(10):4539-47.
13. Markert ML, Hummell DS, Rosenblatt HM, Schiff SE, Harville TO, Williams LW, et al. Complete DiGeorge syndrome: persistence of profound immunodeficiency. J Pediatr. 1998;132(1):15-21.

14. Rota IA, Dhalla F. FOXN1 deficient nude severe combined immunodeficiency. Orphanet J Rare Dis. 2017;12(1):6.

15. Dergousoff BA, Vayalumkal JV, Wright NAM. Survey of infection control precautions for patients with severe combined immune deficiency. J Clin Immunol. 2019;39(8):753-61.

16. Griffith LM, Cowan MJ, Notarangelo LD, Puck JM, Buckley RH, Candotti F, et al. Improving cellular therapy for primary immune deficiency diseases: recognition, diagnosis, and management. J Allergy Clin Immunol. 2009;124(6):1152-60 e12.

17. Rivers L, Gaspar HB. Severe combined immunodeficiency: recent developments and guidance on clinical management. Arch Dis Child. 2015;100(7): 667-72.

18. Rice HE, Skinner MA, Mahaffey SM, Oldham KT, Ing RJ, Hale LP, et al. Thymic transplantation for complete DiGeorge syndrome: medical and surgical considerations. J Pediatr Surg. 2004;39(11): 1607-15.

19. Wong MT, Scholvinck EH, Lambeck AJ, van Ravenswaaij-Arts CM. CHARGE syndrome: a review of the immunological aspects. Eur J Hum Genet. 2015;23(11):1451-9.

20. Cust AE, Armstrong BK, Smith BJ, Chau J, van der Ploeg HP, Bauman A. Self-reported confidence in recall as a predictor of validity and repeatability of physical activity questionnaire data. Epidemiology. 2009;20(3):433-41.

21. Varni JW, Seid M, Kurtin PS. PedsQL 4.0: reliability and validity of the Pediatric Quality of Life Inventory version 4.0 generic core scales in healthy and patient populations. Med Care. 2001;39(8):800-12.

22. Markert ML, Gupton SE, McCarthy E. Experience with cultured thymus tissue in 105 children. Under review. J Allergy Clin Immun. 2021.

23. Markert ML, Alexieff MJ, Li J, Sarzotti M, Ozaki DA, Devlin $\mathrm{BH}$, et al. Postnatal thymus transplantation with immunosuppression as treatment for DiGeorge syndrome. Blood. 2004;104(8):2574-81.

24. McCall RB, Groark CJ, Hawk BN, Julian MM, Merz EC, Rosas JM, et al. Early caregiver-child interaction and children's development: lessons from the St. Petersburg-USA orphanage intervention research project. Clin Child Fam Psychol Rev. 2019;22: 208-24. 
25. Cacioppo JT, Hughes ME, Waite LJ, Hawkley LC, Thisted RA. Loneliness as a specific risk factor for depressive symptoms: cross-sectional and longitudinal analyses. Psychol Aging. 2006;21(1):140-51.

26. Mihalopoulos C, Le LK, Chatterton ML, Bucholc J, Holt-Lunstad J, Lim MH, et al. The economic costs of loneliness: a review of cost-of-illness and economic evaluation studies. Soc Psychiatry Psychiatr Epidemiol. 2020;55(7):823-36.

27. James BD, Wilson RS, Barnes LL, Bennett DA. Latelife social activity and cognitive decline in old age. J Int Neuropsychol Soc. 2011;17(6):998-1005.

28. Collins C, Sharpe E, Silber A, Kulke S, Hsieh EWY. Congenital athymia: genetic etiologies, clinical manifestations, diagnosis, and treatment. J Clin Immunol. 2021. https://doi.org/10.1007/s10875021-01059-7.

29. Mohammadinejad P, Ataeinia B, Kaynejad K, Zeinoddini A, Sadeghi B, Hosseini M, et al. Antibiotic resistance in patients with primary immunodeficiency disorders versus immunocompetent patients. Expert Rev Clin Immunol. 2015;11(10): 1163-72.
30. Goldstein SL, Graham N, Warady BA, Seikaly M, McDonald R, Burwinkle TM, et al. Measuring health-related quality of life in children with ESRD: performance of the generic and ESRD-specific instrument of the Pediatric Quality of Life Inventory (PedsQL). Am J Kidney Dis. 2008;51(2):285-97.

31. Varni JW, Burwinkle TM, Katz ER, Meeske K, Dickinson P. The PedsQL in pediatric cancer: reliability and validity of the Pediatric Quality of Life Inventory Generic Core Scales, Multidimensional Fatigue Scale, and Cancer Module. Cancer. 2002;94(7): 2090-106.

32. Iannaccone ST, Hynan LS, Morton A, Buchanan R, Limbers CA, Varni JW, et al. The PedsQL in pediatric patients with spinal muscular atrophy: feasibility, reliability, and validity of the Pediatric Quality of Life Inventory Generic Core Scales and Neuromuscular Module. Neuromuscul Disord. 2009;19(12):805-12.

33. Joyce $\mathrm{P}, \mathrm{O}^{\prime}$ Rourke $\mathrm{C}$, McDermott $\mathrm{B}$, Heussler $\mathrm{H}$. Health-related quality of life in 22q11.2 deletion syndrome: the child's perspective. J Paediatr Child Health. 2018;54(3):311-5. 